\title{
Modelling of Thermal Behavior of Borehole Heat Exchangers of Geothermal Heat Pump Heating Systems
}

\author{
Gornov V.F. ${ }^{1}$, Peskov N.V. ${ }^{1,2}$, Vasilyev G.P. ${ }^{1}$, Kolesova M.V. ${ }^{1}$ \\ ${ }^{1}$ JSC «INSOLAR-INVEST», Bol'shaya Filevskaya str. 32- 3, Moscow, 121433 Russia \\ ${ }^{2}$ Lomonosov Moscow State University, Leninskie Gory 1, str. 52, Moscow, 119991 Russia
}

\begin{abstract}
This article reports results of comparing the accuracy of the software package "INSOLAR.GSHP.12", modeling non-steady thermal behavior of geothermal heat pump heating systems (GHCS) and of the similar model "conventional" using finite difference methods for solving spatial non-steady problems of heat conductivity. The software package is based on the method of formulating mathematical models of thermal behavior of ground low-grade heat collection systems developed by INSOLAR group of companies. Equations of mathematical model of spatial non-steady thermal behavior of ground mass of lowgrade heat collection system obtained by the developed method have been solved analytically that significantly reduced computing time spent by the software complex "INSOLAR.GSHP.12" for calculations. The method allows to turn aside difficulties associated with information uncertainty of mathematical models of the ground thermal behavior and approximation of external factors affecting the ground. Use of experimentally obtained information about the ground natural thermal behavior in the software package allows to partially take into account the whole complex of factors (such as availability of groundwater, their velocity and thermal behavior, structure and arrangement of ground layers, the Earth's thermal background, precipitation, phase transformations of moisture in the pore space, and more), significantly influencing the formation of thermal behavior of the ground mass of a low-grade geothermal heat collection system. Numerical experiments presented in the article confirmed the high convergence of the results obtained through the software package "INSOLAR.GSHP.12" with solutions obtained by conventional finite-difference methods.
\end{abstract}

\section{Introduction}

Today, geothermal heat pump heating systems (GHCS) are already widely used in temperate regions of the world (North America, Europe, and China [1]). However, practically used mathematical models describing the ground mass thermal behavior in GHCS operation are still far from perfect. Description in models of such complex multi-capillary-porous structures, as the ground, is an extremely difficult task. In the strict sense, in addition to implementation of heat and mass transfer processes, the ground mass thermal behavior models shall take into account the chemical and mineralogical nature of the ground skeleton, mechanical structure of solids material, degree of dispersion of the medium, shape and size of particles and pores, number of phases, quantitative relationships between phases and their mutual arrangement in the medium filling the pore space, as well as many other physical and chemical parameters. Detailed account of these factors in the formulation of a mathematical model is a very serious problem, and its solution often is virtually impossible with the use of modern mathematical apparatus. Analysis of existing models of heat transfer processes in the "ground-BHE" system [2, 3] showed virtual absence of generally accepted algorithms for solving this problem and, in particular, consideration of phase transformations of pore water in the ground.

The software package "INSOLAR.GSHP.12" developed by the Russian company INSOLAR-INVEST allows to simulate the thermal behavior of the ground mass of low-grade geothermal heat collection system in the long-term operation of GHCS [4], taking into account phase transformations of pore water in the ground $[5,6]$.

\section{Mathematical modelling method}

The basis of the software package "INSOLAR.GSHP.12" is the mathematical modelling method based on two fundamental assumptions:

1. Ground mass of a low-grade geothermal heat collection system is treated as quasi-homogeneous body, to which the usual heat conduction equation applies; but with the only difference that heat and mass transfer characteristics are "effective" that allows to partially take into account mass transfer processes in the model.

2. Thermal effect of a ground heat exchanger tube register on the temperature behavior of the ground mass 
of the heat collection system is approximated by introduction of the model of linear heat sinks (sources) of infinitely small diameter, placed in tube locations. This assumption seems justified, since the diameter of tubes of the ground heat exchanger is much less than depth of their laying, whereby the temperature gradient in tube walls can be neglected.

The method is as follows:

Let's consider a ground mass with arbitrarily arranged ground heat exchanger tube register, limited by coordinate planes and parallel to them planes passing through the layers of the ground, non-affected by the heat collection system. In this case, the problem of formulating a mathematical model of such a system leads to the need to address the problem of temperature behavior of the ground mass with heat sinks.

In the most general case, the heat conduction equation, which solution is the unknown temperature of the ground, can be written in the following form:

$$
\mathrm{c} \rho \frac{\partial \mathrm{t}}{\partial \tau}=\frac{\partial}{\partial \mathrm{x}}\left(\lambda \frac{\partial \mathrm{t}}{\partial \mathrm{x}}\right)+\frac{\partial}{\partial \gamma}(\lambda \underset{\partial \gamma}{\partial \gamma})+\frac{\partial \mathrm{t}}{\partial \mathrm{z}}(\lambda \underset{\partial \mathrm{z}}{\mathrm{z}})+\sum_{\mathrm{i}=1}^{\mathrm{n}} \mathrm{q}_{\mathrm{i} \xi \mathrm{i}}
$$

where:

$\mathbf{t}=\mathbf{t}(\mathbf{x}, \boldsymbol{\gamma}, \mathbf{z}, \tau) \quad-\quad$ ground mass unknown temperature, ${ }^{\circ} \mathrm{C}$;

$\mathbf{c} \rho=\mathrm{c} \rho(\mathrm{x}, \gamma, \mathrm{z}, \tau)-\quad$ ground volumetric heat capacity, $\mathrm{J} /\left({ }^{\mathrm{o}} \mathrm{C} \cdot \mathrm{m} 3\right)$;

$\lambda=\lambda(\mathbf{x}, \boldsymbol{\gamma}, \mathbf{z}, \tau, \mathbf{t})-$ ground heat conductivity, $\mathrm{W} /$ $\left(\mathrm{m} \cdot{ }^{\circ} \mathrm{C}\right)$;

$\tau$ - time, sec;

$\mathbf{q}_{\mathrm{i}}=\mathbf{q}_{\mathrm{i}}(\mathbf{x}, \boldsymbol{\gamma}, \mathbf{z}, \tau, \mathbf{t})$ - power of unit of length of the i-th heat sink, W/m;

n - number of heat sinks;

$\xi_{\mathbf{i}}$ - function determining the heat sink position in the ground mass, $1 / \mathrm{m}^{2}$.

The boundary conditions for the equation (1) are as follows:

while $\left\{\begin{array}{rc}x=0 & t=\Psi_{1}(\gamma, z, \tau) ; \\ x=\alpha & t=\Psi_{2}(\alpha, \gamma, z, \\ \gamma=0 & \lambda \stackrel{\partial_{t}}{\tau)}=\alpha \rho(t- \\ \left.t_{n}\right) ; \quad & \begin{array}{c}\partial \gamma \\ \gamma=H\end{array}=\Psi_{3}(x, H, z, \\ \tau) ; \quad t=\Psi_{4}(x, \gamma, 0, \\ \tau=0 \\ \tau) ; z=d \\ \tau) ;\end{array}\right.$

Initial condition

While $\tau=0 \quad t=t_{0}(x, \gamma, z, 0)$;

where:

$\alpha \rho=\alpha_{\rho}(\tau)$ coefficient of heat transfer from the ground surface, $\mathrm{W} /\left(\mathrm{m}^{2} \cdot{ }^{\circ} \mathrm{C}\right)$; $\mathbf{t}_{\mathbf{n}}=\mathbf{t}_{\mathbf{n}} \quad(\boldsymbol{\tau})$ outside air temperature accounting radiation "additive" of the incident solar radiation, ${ }^{\circ} \mathrm{C}$;

$\Psi_{1}, \Psi_{2}, \Psi_{3}, \Psi_{4}, \Psi_{5}$ unknown functions describing temperature at the boundaries of the mass, ${ }^{\circ} \mathrm{C}$;

$\mathbf{t}_{\mathbf{0}}$ initial temperature distribution in the ground, ${ }^{\circ} \mathrm{C}$.

It is obvious that the accuracy of implementation of the resulting mathematical model of the heat collection system thermal behavior will greatly depend on the accuracy of formulation of the boundary conditions (2) and initial temperature distribution $\mathbf{t}_{\mathbf{0}}$, i.e. from the accuracy of approximation of functions $\Psi_{1}, \Psi_{2}, \Psi_{3}, \Psi_{4}$, $\Psi_{5}, \mathbf{t}_{\mathbf{n}}$ and $\mathbf{t}_{\mathbf{0}}$ that, as is known, is a serious problem.

To solve this problem, let's now consider the thermal behavior of the same ground mass, but without heat sinks (sources), i.e. under natural conditions - in the absence of heat effect of the heat collection system on the ground. The heat conductivity equation in this case would look like this:

$$
c * \rho * \frac{\partial t *}{\partial \tau}=\frac{\partial}{\partial x}\left(\lambda * \frac{\partial t *}{\partial x}\right)+\frac{\partial}{\partial \gamma}\left(\lambda * \frac{\partial t *}{\partial \gamma}\right)+\frac{\partial}{\partial z}\left(\lambda * \frac{\partial t *}{\partial z}\right),
$$

where:

* represents a parameter belonging to the problem of natural temperature behavior of the ground mass (without heat sinks or sources), and the other symbols are the same as before.

The boundary conditions in this case can be written as follows:

while $\begin{cases}x=0 & t^{*}=\Psi_{1}(\gamma, z, \tau) ; \\ x=\alpha & t^{*}=\Psi_{2}(\alpha, \gamma, z, \tau) ; \\ \gamma=0 & \lambda^{*} \frac{\partial_{t^{*}}}{\partial \gamma}=\alpha \rho\left(t^{*}-t_{n}\right) ; \\ \gamma=H & t^{*}=\Psi_{3}(x, H, z, \tau) ; \\ z=0 & t^{*}=\Psi_{4}(x, \gamma, 0, \tau) ; \\ z=d & t^{*}=\Psi_{5}(\alpha, \gamma, d, \tau) ;\end{cases}$

Initial condition

While $\tau=0 \quad t^{*}=t_{0}(x, \gamma, z, 0)$;

It should be noted the fact that the coefficient of heat transfer from the ground mass surface $\alpha \rho$ in both problems can be described by the same function $\alpha \rho$ ( $x, \gamma$, $\mathrm{z}, \tau)$, since the heat removal from the ground causes a non-significant change in the surface temperature and therefore has virtually no effect on the rate of heat exchange of the ground surface with the ambient air.

Let's now consider the difference of the resulting equation systems, that is lets subtract the system of equations (1)-(6) describing the thermal behavior of the ground mass with heat sinks (sources) from the system (1)-(4), describing the thermal behavior of the ground mass in its natural state (without heat sinks (sources)).

When subtracting the equation (1) from the equation (4) we obtain

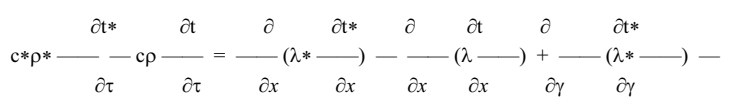


$-\frac{}{\partial \gamma}(\lambda \underset{\partial \gamma}{\longrightarrow})+\frac{-}{\partial z}\left(\lambda^{*} \underset{\partial z}{-}\right)-\frac{-}{\partial z}(\lambda \underset{\partial z}{\longrightarrow})+\sum_{\mathrm{i}=1} \mathrm{q}_{\mathrm{i} \xi} \mathrm{i}$.

Subtracting the boundary conditions (2) from the boundary conditions (4) gives

while $\left\{\begin{array}{cc}x=0 & t^{*}-t=0 ; \\ x=\alpha & t^{*}-t=0 ; \\ \gamma=0 & \lambda^{*}-\partial_{t^{*}}-\lambda^{*} \frac{\partial_{t}}{}=\alpha \rho\left(t^{*}-\right. \\ \gamma=H & t^{*}-t=0 ; \\ z=0 & t^{*}-t=0 ; \\ z=d & t^{*}-t=0 ;\end{array}\right.$

Subtracting the initial conditions of the system (2) from the initial conditions (5) gives while

$$
\tau=\mathbf{0} \quad \mathbf{t}^{*}-\mathbf{t}=\mathbf{0} .
$$

Let's introduce a new symbol

$$
\mathbf{T}=\mathbf{t}^{*}-\mathbf{t}
$$

and, substituting variables in the system (7) - (9) taking into account this symbol, we obtain a new equation $^{1}$ ):

$$
\begin{aligned}
& c \rho \frac{\partial T}{\partial \tau}=\frac{\partial}{\partial x}\left(\lambda \frac{\partial T}{\partial x}\right)+\frac{\partial}{\partial \gamma}\left(\lambda \frac{\partial T}{\partial \gamma}\right)+\frac{\partial}{\partial z}\left(\lambda \frac{\partial T}{\partial z}\right)+\sum_{i=1}^{n} q_{i} \xi_{i}+ \\
& \left\{\frac{\partial}{\partial x}\left[\left(\lambda^{*}-\lambda\right) \frac{\partial t^{*}}{\partial x}\right]+\frac{\partial}{\partial \gamma}\left[\left(\lambda^{*}-\lambda\right) \frac{\partial t^{*}}{\partial \gamma}\right]+\frac{\partial}{\partial z}\left[\left(\lambda^{*}-\lambda\right) \frac{\partial t^{*}}{\partial z}\right]-\right. \\
& \left.\left(c^{*} \rho^{*}-c \rho\right) \frac{\partial t^{*}}{\partial \tau}\right\}
\end{aligned}
$$

$$
\begin{aligned}
& \begin{array}{llllllll}
\partial \mathrm{T} & \partial & \partial \mathrm{T} & \partial & \partial \mathrm{T} & \partial & \partial \mathrm{T} & \mathrm{n}
\end{array} \\
& \mathrm{c} \rho-=-(\lambda \longrightarrow)+\longrightarrow(\lambda \longrightarrow)+\longrightarrow(\lambda \longrightarrow)+\sum \mathrm{q}_{\mathrm{i} \xi \mathrm{i}} \\
& \begin{array}{llllllll}
\partial \tau & \partial \mathrm{x} & \partial \mathrm{x} & \partial \gamma & \partial \gamma & \partial \mathrm{z} & \partial \mathrm{z} & \mathrm{i}=1
\end{array}
\end{aligned}
$$

Let's make a similar substitution of variables in the boundary conditions (8):

while $\left\{\begin{aligned} x & =0 \quad T=0 ; \\ x=0 \quad & =\propto \quad T=0 ; \\ \lambda \frac{\partial y}{\partial x} & =\alpha \rho T-\left(\lambda^{*}-\lambda\right) \frac{\partial t^{*}}{\partial \gamma} \\ \gamma & =H \quad T=0 ; \\ z & =0 \quad T=0 ; \\ z & =d \quad T=0 .\end{aligned}\right.$

A similar substitution of variables in the initial conditions (9) allows to obtain

while

$$
\tau=\mathbf{0} \quad \mathbf{T}=\mathbf{0} .
$$

Now let's consider in more detail the resulting boundary conditions (11). As discussed earlier, the

1 Hereinafter, it should be understood that similar substitution of variables has been performed in functions c, $\rho$ and $\lambda$ as well. ground masses addressed in the problems are limited by conventional planes, which arrangement eliminates the heat effect of ground heat exchanger on the boundary ground layers. Consequently, the ground temperatures on the boundaries of the mass, except only the surface temperatures in the general case, will be practically identical in both problems. Accordingly, heat conductivities of ground masses considered in problems will be equal as well. As a result, without reducing the model accuracy, we can conclude that the difference of ground heat conductivities $\left(\lambda^{*}-\lambda\right)$ for all the boundaries of the mass, including in this case (for heat collection systems) the ground surface is equal to 0 , i.e. $\lambda *-\lambda=\mathbf{0}$.

In view of this point, the boundary conditions (13) will be

$$
\text { while }\left\{\begin{array}{c}
x=0 \quad T=0 \\
x=\alpha \quad T=0 \\
\gamma=0 \quad \lambda \frac{\partial T}{\partial \gamma}=\alpha \rho T \\
\gamma=H \quad T=0 \\
z=0 \quad T=0 \\
z=d \quad T=0
\end{array}\right.
$$

Thus, as a result of these transformations we have obtained a new mathematical model in which the required function is unknown function $\mathrm{T}$, which is function of effect of heat sinks on the natural thermal behavior of the ground and equal to temperature difference of ground masses in a natural condition and with heat sinks (sources). It should be noted that in the boundary conditions of the new model (the boundary conditions (15) and the initial condition (14)) we managed to dispense with unknown functions $\psi_{1}, \psi_{2}, \psi_{3}, \psi_{4}, \psi_{5}$ and unknown initial ground temperature distribution, as well as the need for approximation of the annual change of the ambient air temperature and the intensity of solar radiation incident on the surface of the ground mass under consideration. However, the equation (11) has unknown function $t^{*}$, which is the solution of the "base" problem, describing the natural thermal behavior of the ground. Naturally, the accuracy of formulating this function will have the most significant effect on the accuracy of implementation of the resulting mathematical model and its adequacy to real processes occurring in the ground.

In general, the "base" problem solution can be formulated in the model with the use of hydrometeorological data on the annual change of ground temperatures at different depths, which are monitored by virtually all weather stations.

Thus, use of the method described in the formulation of mathematical models of thermal behavior of ground low-grade heat collection systems allows to turn aside difficulties associated with approximation of external factors affecting the system. A definite advantage of this method compared with the traditional approach to modelling of thermal processes occurring in such systems is the fact that the use of experimentally obtained information about the natural ground thermal behavior in the model allows to partially take into account the whole 
complex of factors (such as availability of groundwater, and their velocity and thermal behavior, structure and arrangement of ground layers, the Earth's thermal background, precipitation, phase transformations of moisture in the pore space, and more), significantly influencing the formation of thermal behavior of the heat collection system, which joint account in a strict formulation of the problem is practically impossible now.

Furthermore, it should be noted that, if necessary, this method allows to use data obtained as a result of theoretical studies of the natural thermal behavior of the considered ground mass as the "base" problem solution.

If necessary, the reverse transition from the function of heat sinks effect on the natural behavior conditions of the ground mass to actual temperatures observed in the mass in the course of the heat collection system operation is carried out using substitution (10).

This method of mathematical modelling of non-steady thermal behavior of ground low-grade heat collection systems has been implemented in the software package "INSOLAR.GSHP.12", providing determination of optimal parameters of a heat collection system depending on the climatic conditions of the construction area, heatprotective characteristics of the building, performances of heat pumps, circulating pumps, heating devices, as well as their modes of operation. Use of this method of mathematical modelling in thee software package "INSOLAR.GSHP.12" allowed to turn aside difficulties associated with informative uncertainty of models and approximation of external climatic effects on ground mass by using the experimentally obtained information about the natural ground thermal behavior, which allows to partially take into account the whole complex of factors (such as availability of groundwater, their velocity and thermal behavior, structure and arrangement of ground layers, the Earth's thermal background, precipitation, phase transformations of moisture in the pore space, and more), significantly influencing the formation of thermal behavior of the heat collection system, which joint account in a strict formulation of the problem is practically impossible now.

\section{Accuracy assessment}

To assess the accuracy of the software package "INSOLAR.GSHP.12" we compared the results of calculations performed by traditional software packages using numerical methods for modelling of thermal behavior of processes occurring in the ground, and the calculation results obtained by the software package "INSOLAR.GSHP.12".

The comparative assessment solved the same problem in both cases: the thermal behavior of the ground mass with intermittent point linear heat sink horizontally laid at a certain depth from the earth surface was considered. At the same time, the effect of a heat sink on a heat field of the surrounding ground was assessed. The sink operated periodically: it was enabled at the time zero, operated 7 months, and then it was disabled and restarted by the 2nd-5th year.
The calculations were carried out for the ground mass in the form of a parallelepiped with dimensions of $100 \times 100 \times 300 \mathrm{~m}(\mathrm{HxWxL})$ with a linear heat sink in the length of $70 \mathrm{~m}$ laid at a depth of $30 \mathrm{~m}$ from the surface. The sink operated periodically: it was enabled on October 1 (time zero) and operated 7 months (till April 30), then it was disabled and restarted by the second year from 13 to 19 months and so on during 5 years.

The calculations used the following thermal characteristics of the ground: heat conductivity -2.65 $\mathrm{W} /(\mathrm{m} * \mathrm{C})$; heat capacity $-0.47 \mathrm{Wh} /(\mathrm{kg} * \mathrm{C})$; density $2000.00 \mathrm{~kg} /$ cu.m. For all boundaries, homogeneous boundary conditions were formulated. Heat flow was assumed to be zero at all boundaries except the ground mass' surface. On the ground surface the mass lost heat to the environment at a constant temperature of the outside air - the boundary condition of the third kind. The coefficient of heat transfer from the ground surface was assumed to be $23.26 \mathrm{~W} /$ (sq.m). The initial temperature of the ground around the mass was equal to zero.

The heat sink was a tube heat exchanger with a radius of $0.08 \mathrm{~m}$. Cooling was provided by a coolant that was pumped through the heat exchanger. The coolant heat capacity was adopted equal to $1.05 \mathrm{~W} * \mathrm{~h} /\left(\mathrm{kg}^{*} \mathrm{oC}\right)$, and its density was equal to $1020.00 \mathrm{~kg} / \mathrm{cu} . \mathrm{m}$.

In the traditional problem solved by numerical methods based on the symmetry condition, the half of the computational domain was considered. The number of grid node was assumed to be 33177 , the number of cells (tetrahedrons) was equal to 157845 . The computational grid was created using the software Gmsh (http://geuz.org/gmsh/). The heat sink power was $5 \mathrm{~kW}$ for the entire ground heat exchanger. [7]

Figs. 1-3 show the calculation model and results of calculation according to the conventional version of calculation using numerical methods, and Fig. 4 shows the results of calculations obtained by the software complex "INSOLAR.GSHP.12".

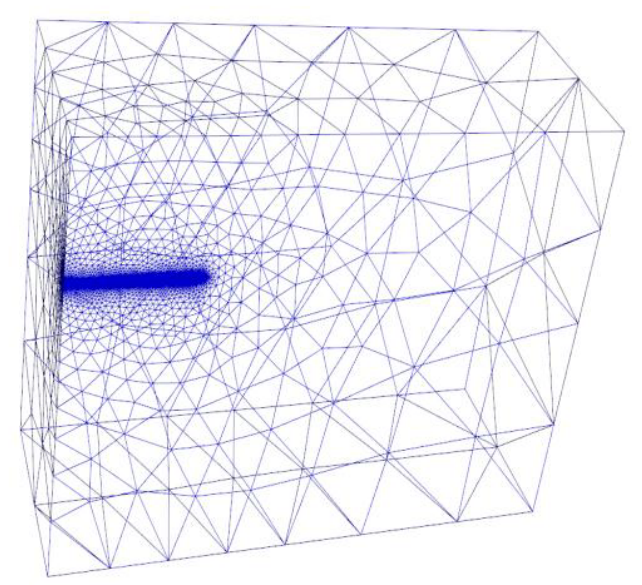

Figure 1. Computational domain (computational grid)

Comparison of charts of coolant temperature variation in the ground heat collection system shown in Figs. 3 and 4 clearly demonstrate a high convergence of the results with those obtained by conventional numerical methods, 
and confirm the accuracy of the mathematic models underlying the software package "INSOLAR.GSHP.12".

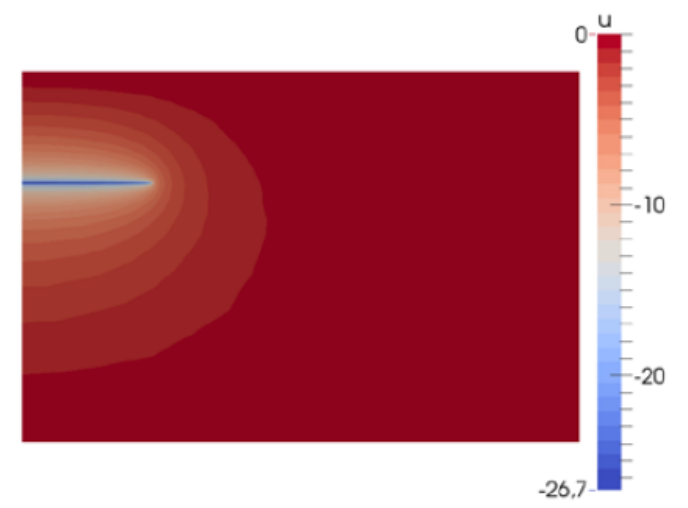

Figure 2. Temperature field in the ground mass (vertical section across the heat exchanger)

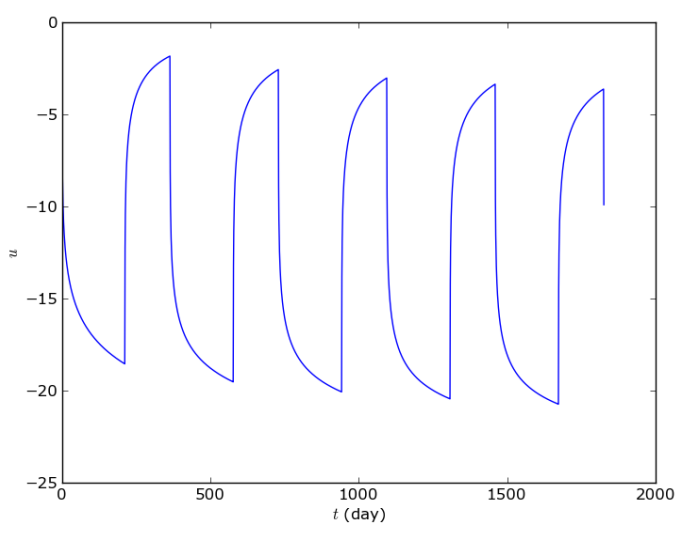

Figure 3. Ground temperature at the heat sink point

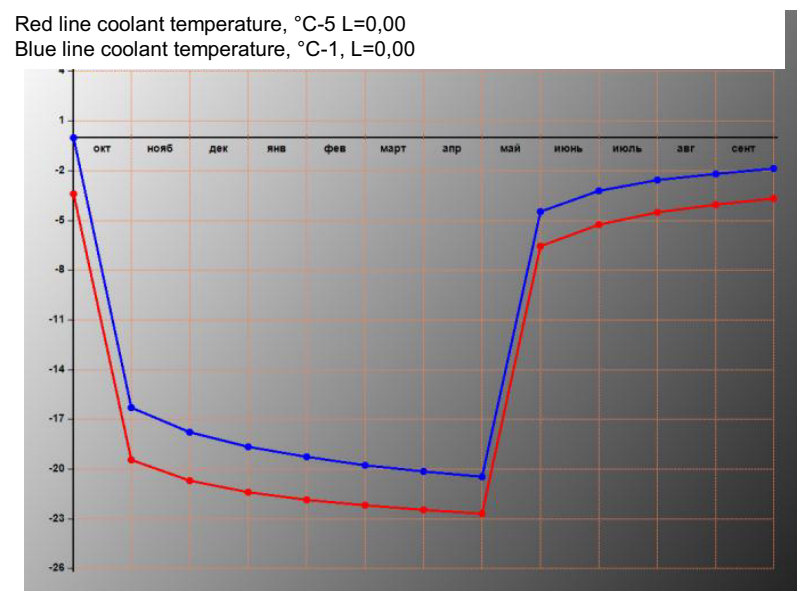

Figure 4. The coolant temperature at the heat sink point (1st (blue curve) and 5th (red curve) years of operation), calculated using the software package "INSOLAR.GSHP.12"

\section{Conclusion}

Results of numerical experiments presented in the article confirmed the high convergence of mathematical models underlying the software package "INSOLAR.GSHP.12" with "conventional" numerical models. Use of the mathematical modelling method described in the article allowed to describe the spatial non-steady thermal behavior of the ground mass of a low-grade geothermal heat collection system using a second-order differential equation system with homogeneous boundary conditions. The solution of this differential equation system was obtained by the analytical method of finite integral transformations. Use of analytical solution significantly reduced the "computing" time required for calculations, which in turn allowed to create the software package "INSOLAR.GSHP.12" with advanced features. The software package "INSOLAR.GSHP.12" actually solves the problem of multiparametric optimization of GHCS configuration for the specific building and construction area. At the same time the objective function of the optimization problem is a minimum annual energy costs for GHCS operation, and optimization criteria are radius of tubes of a ground heat exchanger, and its (heat exchanger) length and depth.

\section{Acknowledgements}

The research was conducted by JSC "INSOLARINVEST" with the financial support of the Ministry of Education and Science of Russia. Unique identifier of the project RFMEFI57915X0115.

\section{References}

1. Lund J.W., Freeston D.H., Boyd T.L. Direct utilization of geothermal energy 2010 worldwide review. Geothermics, 40, 159-180, (2011).

2. Yang H., Cui P., Fang Z. Vertical-borehole groundcoupled heat pumps: A review of models and systems, Applied Energy, 87, 16-27, (2010).

3. Lamarche L., Kajl S., Beauchamp B. A review of methods to evaluate borehole thermal resistances in geothermal heat-pump systems. Geothermics, 39, 187-200, (2010).

4. G.P. Vasilyev, N.V. Peskov, M.M. Brodach, V.A. Lichman, A.N. Dmitriev,V.F. Gornov, M.V. Kolesova, "Estimation of the thermal effect of ground moisture condensation on heat transfer outside a geothermal borehole", Energy and Buildings 82, 795-798, (2014).

5. G. P. Vasilyev, N. V. Peskov, V. A. Lichman, V. F. Gornov, M. V. Kolesova, «Simulating the thermal operating conditions in the thermal wells of ground source heat pump heat supply systems. part I: accounting the porous moisture freezing processes in soil» Thermal Engineering, (2015), 62(8), pp. 547552.

6. Carslaw H.S., Jaeger J.C. Conduction of heat in solids. Oxford UK: Claremore Press, (1946).

7. Ingersoll L.R., Zobel O.J., Ingersoll A.C. Heat conduction with engineering, geological, and other applications. New York: McGraw-Hill, (1954). 